\title{
Burden of Alcohol Abuse or Dependence Among Long-Term Opioid Users with Chronic Noncancer Pain
}

\author{
Pamela B. Landsman-Blumberg, MPH, DrPH; Nathaniel Katz, MD, MS; Kavita Gajria, MS; \\ Anna D. Coutinho, BPharm, PhD; Paul P. Yeung, MD, MPH; and Richard White, PhD, MPH, MBE
}

\begin{abstract}
BACKGROUND: Substance abuse disorders among chronic noncancer pain (CNCP) patients add to the clinical challenges and economic burden of caring for such patients. Despite potential risks, some CNCP patients with a history of alcohol abuse or dependence (AAD) and pain that is refractory to nonopioid treatment options may still need opioids for pain management. However, there is a lack of data on adverse outcomes in long-term opioid users with CNCP and a history of substance abuse or AAD disorders.
\end{abstract}

OBJECTIVE: To compare adverse outcomes and all-cause health care costs among CNCP patients on long-term opioid treatment with and without a previous diagnosis of AAD.

METHODS: Using MarketScan claims databases (2006-2012), CNCP patients with $\geq 90$ days of opioid supply after CNCP diagnosis and continuous enrollment 12 months before CNCP diagnosis (baseline period) and 12 months after opioid start (post-index period) were identified. AAD was defined by diagnosis codes at any time before opioid initiation. Outcomes included opioid overdose, accident, and injury episodes identified by ICD-9-CM diagnoses codes. T-tests and Mann-Whitney tests compared continuous measures, and chi-square and Fisher's exact tests compared categorical measures between those with and without AAD. Multivariable analyses for outcomes were conducted, adjusting for baseline differences between cohorts.

RESULTS: Of 21,203 CNCP patients with long-term opioid treatment, 750 (3.5\%) had an AAD diagnosis before opioid initiation. AAD patients were significantly younger (48.4 [SD \pm 11.4$]$ years vs. $52.8[S D \pm 14.8]$ years), less likely to be enrolled in Medicare (17\% commercial vs. $4 \%$ Medicare), and more likely to be male $(67 \%$ vs. $48 \%$; all $P<0.001)$. There were no differences in type or number of CNCP diagnoses or Charlson Comorbidity Index (CCI) scores. Patients with AAD had significantly higher rates of depression and anxiety diagnoses, antidepressant and benzodiazepine use, and drug abuse/dependence diagnoses in the baseline period. Twelvemonth post-index rates of opioid overdose $(1.2 \%$ vs. $0.2 \%)$, accident $(7.3 \%$ vs. $2.8 \%$ ), and injury ( $46.1 \%$ vs. $36.8 \%$ ) were greater in the AAD cohort (all $P<0.001)$. The differences were nonsignificant for accidents in multivariable analyses. While mean prescription costs were similar $(\$ 3,562$ vs. $\$ 3,312 ; P=0.212)$, AAD patients had significantly higher mean all-cause medical costs $(\$ 28,429$ vs. $\$ 22,082 ; P<0.001)$ and significantly higher all-cause total health care costs $(\$ 31,991$ vs. $\$ 25,395 ; P<0.001)$. The cost differences remained significant in multivariable analyses.

CONCLUSIONS: In the first year after long-term opioid initiation, CNCP patients with a previous AAD diagnosis had 5 times the rate of opioid overdose, 2.3 times the rate of accidents, 1.2 times the rate of injury, and higher all-cause health care costs compared with those not diagnosed with AAD.

J Manag Care Spec Pharm. 2017;23(7):718-24

Copyright $\odot 2017$, Academy of Managed Care Pharmacy. All rights reserved.

\section{What is already known about this subject}

A history of alcohol abuse or dependence (AAD) is considered a strong predictor of potential opioid abuse or misuse and may be considered a relative contraindication to the use of opioids in chronic noncancer pain (CNCP) patients.

Opioids may be a necessary pain relief treatment option among patients with a history of AAD and who have CNCP that is refractory to nonopioid treatments.

There are limited published data available to describe the adverse outcomes of long-term opioid use in patients with CNCP and a history of AAD.

\section{What this study adds}

In this cohort of patients with $\mathrm{CNCP}$ and long-term opioid use, the risks of overdose, accident, or injury were higher among patients with a history of AAD compared with those without AAD.

During the 1 year following the initiation of long-term opioid therapy, CNCP patients with a history of AAD had higher health care costs than those without a history of AAD.

The risks and benefits of treating patients with a history of AAD with long-term opioid therapy must be carefully considered.

$\square$ ubstance abuse disorders are common among chronic noncancer pain (CNCP) patients, adding to the clinical challenges and economic burden of caring for such patients. The reported prevalence of substance abuse disorders among CNCP patients ranges from 3\% to $48 \%{ }^{1}$. The use of opioids, a common treatment modality for CNCP patients, further compounds the burden of substance abuse in these patients.

Previous research has found long-term CNCP opioid users to be significantly more likely to have been diagnosed with alcoholism and nonopioid drug abuse (e.g., antidepressants, sedatives) compared with CNCP patients not using opioids. ${ }^{2-4}$ A history of alcohol abuse is also considered a strong predictor of potential for opioid abuse and misuse. ${ }^{5}$ The concomitant use of alcohol and opioids relative to using either alone increases the abuse liability effects such as drug liking and the desire to take the drug again. ${ }^{6}$ Consequently, a history of alcohol abuse may be considered a relative contraindication to opioid use in CNCP patients. ${ }^{7}$ However, some CNCP patients with a 


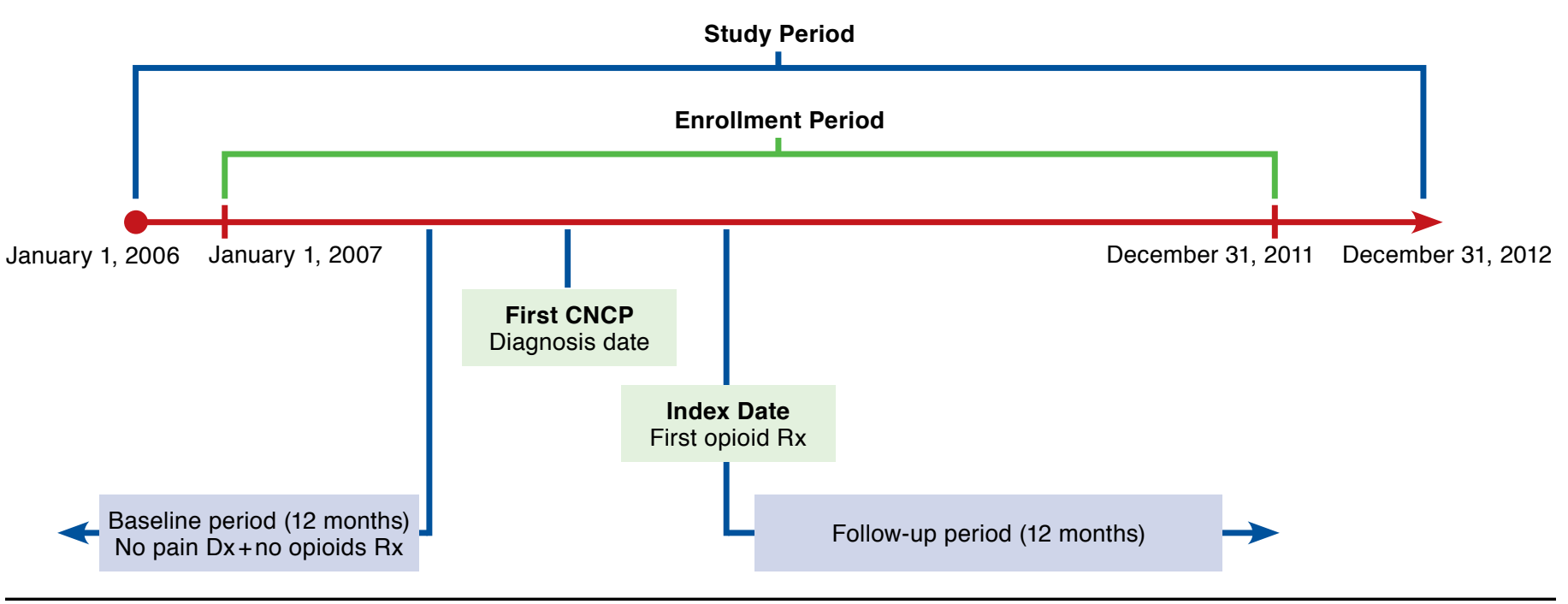

history of alcohol abuse and pain that is refractory to nonopioid treatment options may still need opioids for pain management once an appropriate assessment of the risk and benefit of such therapies is done.

There is a paucity of information in the published literature on adverse outcomes in long-term opioid users with CNCP and history of substance abuse disorder. Weisner et al. (2009) observed that, among CNCP patients on long-term opioid therapy, those with substance abuse disorder received higher doses and more days supply of opioids compared with those without substance abuse disorder. ${ }^{8}$ However, clinical outcomes and nonopioid health care utilization were not assessed. A small case series in patients with a history of opioid abuse receiving opioids (any duration) for noncancer pain found an association between history of opioid abuse and aberrant behaviors; again, clinical and utilization outcomes were not assessed. ${ }^{9}$ The objective of the current study was to compare rates of adverse outcomes such as opioid overdose, accident, and injury and health care costs between CNCP long-term opioid users with and without diagnosed alcohol abuse or dependence (AAD).

\section{Methods}

\section{Study Design}

This study was a retrospective cohort analysis of patients with CNCP and long-term opioid use. CNCP was defined as having at least 3 nondiagnostic medical claims 1 to 12 months apart with International Classification of Diseases, Ninth Revision, Clinical Modification (ICD-9-CM) codes of the same pain type (back, neck, osteoarthritis, fibromyalgia, or neuropathic pain) in any diagnosis position (primary or secondary) between January 1, 2007, and December 31, 2011. Nondiagnostic medical claims refer to all medical claims except those for services provided in the laboratory or radiology setting. These data were used in the identification of chronic pain to increase the probability that the service was not to rule out a diagnosis but to treat the disease.

The index date was defined as the date of the first opioid prescription fill (Figure 1). Long-term opioid therapy was defined as at least 90 days supply of any Schedule II or III/IV opioid within the first 6 months after the index date. A 12-month follow-up period, beginning on the index date, was used to evaluate all study outcomes. A 12-month baseline period before the first CNCP diagnosis date was used to assess demographic and clinical characteristics of the study sample.

\section{Data Source}

Integrated medical and prescription claims data (January 2006December 2012) from the Truven Health MarketScan Commercial Claims and Encounters Database (commercial) and Medicare Supplemental and Coordination of Benefits Database (Medicare supplemental) were used. Both databases contain inpatient and outpatient medical and prescription claims with information on diagnoses, procedures, prescription fills, dates of service, and person-level enrollment information. The commercial database includes over 40 million enrollees annually, representing employees, their spouses, and dependents covered by employer-sponsored private health insurance. Health care for these individuals is provided under 


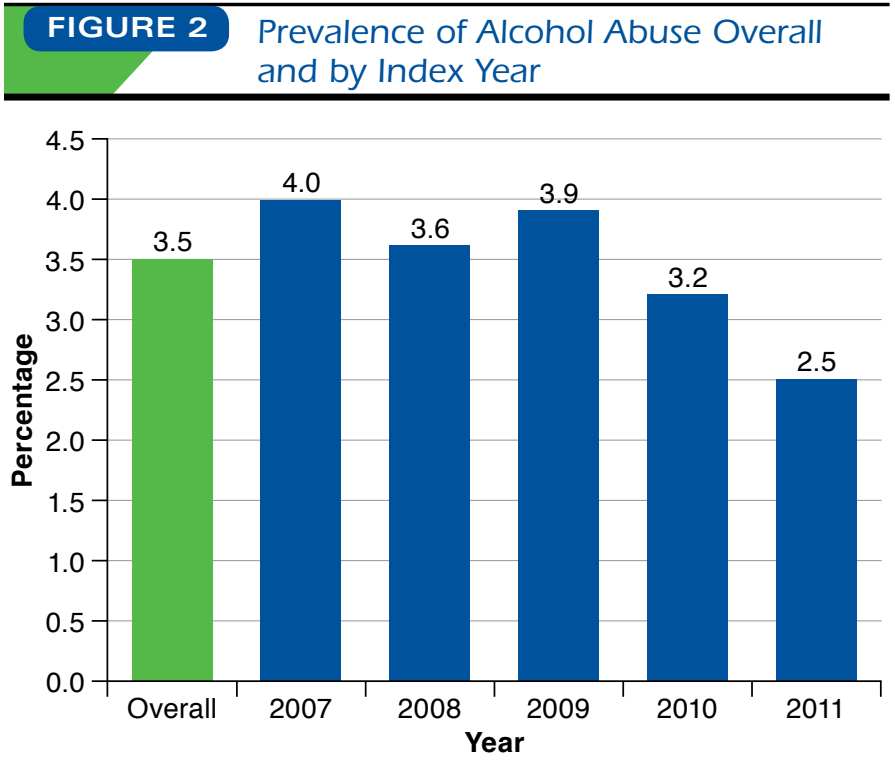

a variety of fee-for-service, fully capitated, and partially capitated health plans, including preferred and exclusive provider organizations, point-of-service plans, and health maintenance organizations. The Medicare supplemental database includes approximately 4.3 million Medicare-eligible persons annually enrolled in supplemental insurance plans offered by their former employers. The Medicare-covered portion of payment is captured as the coordination of benefits amount. Patients can be followed longitudinally and as they move from the commercial to the Medicare supplemental database through the use of a unique blinded identifier.

\section{Study Cohorts and Criteria}

Long-term opioid users with $\mathrm{CNCP}$ were classified into 2 cohorts: those with and those without a diagnosis of AAD. AAD was defined as the presence of at least 1 medical claim with a primary or secondary ICD-9-CM diagnosis code of 303.x or 305.0x at any time during the baseline period before the index date.

Patients were required to be aged 18 years or older on the first $\mathrm{CNCP}$ diagnosis date; to have no pain diagnosis and no prescription claim for an opioid in the 12 months before the first pain diagnosis date; to have no diagnosis of a malignancy any time in the 12 months preceding or any time following the first pain diagnosis date; and to be continuously enrolled in medical and pharmacy benefit plans during the 12 months before the first pain diagnosis date and the 12 months following the index date. Patients without a prescription claim for any Schedule II or III/IV opioids or $<90$ days supply of opioids (short-term users) were excluded.

\section{Outcomes}

Study outcomes included adverse outcomes and all-cause health care costs. Adverse outcomes included opioid overdose, accident, and injury that were readily identified using ICD-9-CM diagnosis codes. All-cause health care costs (standardized to 2012 U.S. dollars) were also evaluated and computed using payments received by providers of care for medical and prescription claims, regardless of diagnosis code, including payments from insurers and patients (i.e., copayments, coinsurance, and deductibles). All-cause medical costs (inpatient and outpatient), prescription costs, and total costs (medical and prescription) were calculated in the 12 months post-index.

\section{Statistical Analysis}

Descriptive statistics (means and proportions) were used to characterize the study sample during the baseline period. Patient characteristics assessed at baseline included age, gender, region, Charlson Comorbidity Index (CCI) score, ${ }^{10}$ urban or rural residence, health plan type, primary payer, index year, type of CNCP diagnoses, number of CNCP diagnoses, baseline comorbid conditions, and baseline use of pain-related prescription medications.

All binary and categorical outcomes were presented as proportions, and chi-square or Fischer's exact tests were used as appropriate to assess differences between long-term opioid users with and without AAD. Means, standard deviations, and medians (as appropriate) were presented for continuous outcomes, and t-tests or Mann-Whitney tests were used to assess unadjusted differences between long-term opioid users with and without AAD. Multivariable logistic models were used to assess differences in odds of adverse events between opioid users with and without AAD.

Multivariable analyses using generalized linear models with log-link function and gamma distribution were used to assess differences in total health care costs and cost components (medical and prescription) between opioid users with and without AAD. All models controlled for demographic and clinical characteristics assessed at baseline. Adjusted average costs with 95\% confidence intervals for total, medical, and prescription utilization are presented. All models controlled for difference in baseline characteristics except for baseline rate of nonopioid drug abuse due to multicollinearity with AAD.

\section{Results}

A total of 21,203 patients were included in the final sample, of which $3.5 \%$ had an AAD diagnosis (Appendix, available in online article). The annual baseline rate of diagnosed AAD among long-term opioid users with CNCP declined from 4.0\% in 2007 to $2.5 \%$ in 2011 (Figure 2). At baseline, long-term opioid users with AAD were on average approximately 4 years 


\begin{tabular}{|c|c|c|c|c|c|}
\hline \multirow{2}{*}{$\begin{array}{l}\text { Characteristic } \\
\text { Age, years, mean (SD) }\end{array}$} & \multicolumn{2}{|c|}{$\begin{array}{l}\text { With AAD } \\
\mathrm{n}=750\end{array}$} & \multicolumn{2}{|c|}{$\begin{array}{c}\text { Without AAD } \\
\mathbf{n}=20,453\end{array}$} & \multirow{2}{*}{$\frac{P \text { Value }^{\mathrm{a}}}{<0.001}$} \\
\hline & 48.4 & $(11.4)$ & 52.8 & $(14.8)$ & \\
\hline Male, n (\%) & 499 & $(67)$ & 9,841 & (48) & $<0.001$ \\
\hline \multicolumn{6}{|l|}{ Residence type, n (\%) } \\
\hline Urban & 597 & $(80)$ & 15,853 & $(78)$ & \multirow{3}{*}{0.046} \\
\hline Rural & 138 & (18) & 4,341 & $(21)$ & \\
\hline Unknown & 15 & (2) & 259 & (1) & \\
\hline \multicolumn{6}{|c|}{ Geographic region, $\mathbf{n}(\%)$} \\
\hline Northeast & 103 & (14) & 2,295 & (11) & \multirow{5}{*}{$<0.001$} \\
\hline North Central & 238 & (32) & 6,112 & $(30)$ & \\
\hline South & 240 & (32) & 8,454 & (41) & \\
\hline West & 154 & (21) & 3,330 & $(16)$ & \\
\hline Unknown & 15 & $(2)$ & 262 & (1) & \\
\hline \multicolumn{6}{|l|}{ Payer, n (\%) } \\
\hline Commercial & 717 & (96) & 17,021 & (83) & \multirow{2}{*}{$<0.001$} \\
\hline Medicare & 33 & (4) & 3,432 & (17) & \\
\hline \multicolumn{6}{|c|}{ Type of chronic pain diagnosis, ${ }^{b}$ n (\%) } \\
\hline Low back & 644 & $(91)$ & 17,584 & $(90)$ & 0.554 \\
\hline Neck & 350 & $(49)$ & 10,013 & $(51)$ & 0.304 \\
\hline Osteoarthritis & 325 & $(48)$ & 9,022 & $(46)$ & 0.828 \\
\hline Fibromyalgia & 147 & $(21)$ & 4,436 & (23) & 0.210 \\
\hline Neuropathic pain & 237 & (33) & 5,988 & $(31)$ & 0.122 \\
\hline \multicolumn{6}{|c|}{ Number of chronic pain diagnoses, $\mathbf{n}(\%)$} \\
\hline 1 & 511 & $(68)$ & 13,844 & $(68)$ & \multirow{3}{*}{0.838} \\
\hline 2 & 200 & $(27)$ & 5,441 & $(27)$ & \\
\hline$\geq 3$ & 39 & (5) & 1,168 & (6) & \\
\hline \multicolumn{6}{|l|}{ Comorbidities, n (\%) } \\
\hline Depression & 201 & $(27)$ & 2,668 & (13) & $<0.001$ \\
\hline Anxiety & 117 & $(16)$ & 1,852 & (9) & $<0.001$ \\
\hline Sleep disturbances & 77 & $(10)$ & 1,926 & (9) & 0.434 \\
\hline Hypertension & 301 & $(40)$ & 8,149 & $(40)$ & 0.873 \\
\hline $\begin{array}{l}\text { Other cardiovascular } \\
\text { disease }\end{array}$ & 116 & (15) & 2,877 & (14) & 0.279 \\
\hline Ischemic heart disease & 37 & (5) & 1,894 & (9) & $<0.001$ \\
\hline Diabetes & 85 & (11) & 3,336 & (16) & $<0.001$ \\
\hline $\begin{array}{l}\text { Drug abuse and/or } \\
\text { dependence }\end{array}$ & 66 & (9) & 354 & (2) & $<0.001$ \\
\hline CCI, mean (SD) & 0.70 & (1.3) & 0.70 & $(1.1)$ & 0.060 \\
\hline \multicolumn{6}{|c|}{ Concomitant medications, $\mathrm{n}(\%)$} \\
\hline NSAIDs/acetaminophen & 285 & (38) & 8,681 & $(42)$ & 0.016 \\
\hline Muscle relaxants & 243 & (32) & 6,948 & (34) & 0.372 \\
\hline Corticosteroids & 147 & (20) & 4,672 & (23) & 0.037 \\
\hline Antidepressants & 292 & (39) & 6,156 & (30) & $<0.001$ \\
\hline Anticonvulsants & 151 & $(20)$ & 3,893 & (19) & 0.452 \\
\hline Benzodiazepines & 197 & $(26)$ & 3,947 & (19) & $<0.001$ \\
\hline \multicolumn{6}{|c|}{ 12-month baseline all-cause costs (\$), mean (SD) } \\
\hline Total cost & 7,077 & $22,186)$ & 9,361 & $(21,308)$ & 0.0056 \\
\hline Medical cost & 5,299 & $21,648)$ & 7,611 & $(20,552)$ & 0.0040 \\
\hline Prescription cost & 1,778 & $(3,461)$ & 1,749 & $(4,172)$ & 0.8249 \\
\hline \multicolumn{6}{|c|}{$\begin{array}{l}\text { Note: Bold font indicates } P<0.05 \text { and statistical significance. } \\
{ }^{a} T \text {-test for continuous variables and chi-square test for categorical variables. } \\
\text { bTypes of chronic pain are nonmutually exclusive categories; first recorded chronic } \\
\text { pain diagnoses are mutually exclusive categories. } \\
\text { AAD=alcohol abuse or dependence; CCI=Charlson Comorbidity Index; } \\
\text { NSAID=nonsteroidal anti-inflammatory drug; } S D=\text { standard deviation. }\end{array}$} \\
\hline
\end{tabular}

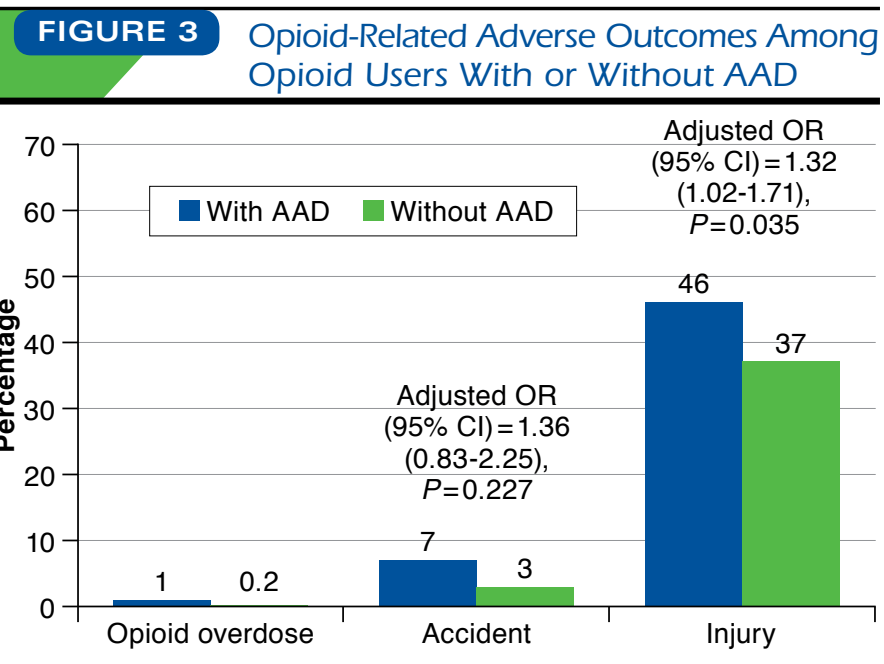

Note: All unadjusted prevalence rate comparisons $P<0.001$ (chi-square test). Adjusted OR could not be obtained for opioid overdose because of very low prevalence rates.

$A A D=$ alcohol abuse or dependence; $C I=$ confidence interval; $O R=$ odds ratio.

younger ( 48.4 years vs. 52.8 years; $P<0.001$ ) and more likely to be male $(67 \%$ vs. $48 \% ; P<0.001)$ than patients without an AAD diagnosis (Table 1). Regardless of AAD status, the majority of patients were diagnosed with low back pain. Neck pain and osteoarthritis were each diagnosed in approximately half of the CNCP population. Two thirds of patients in both cohorts had claims diagnoses for only 1 type of CNCP.

While the average total comorbidity burden represented by the CCI score was similar between the study cohorts $(0.70$ for each; $P=0.060$ ), a significantly greater proportion of opioid users with AAD were diagnosed with the specific comorbidities of depression and anxiety before their CNCP diagnoses compared with those without AAD (Table 1; $P<0.001$ for each). Correspondingly, the use of antidepressants and benzodiazepines was also significantly higher among the AAD cohort $(P<0.001$ for both). A significantly greater proportion of patients with AAD were also diagnosed with drug abuse and/or dependence $(P<0.001)$ during the baseline period. Ischemic heart disease and diabetes were less frequently diagnosed among long-term opioid users with AAD compared with those users without AAD $(P<0.001$ for both).

During the 12-month baseline period, all-cause total costs were $\$ 2,284$ lower for the AAD cohort versus the cohort without AAD (Table 1; $P<0.05$ ). This was driven by all-cause medical costs, which were $\$ 2,312$ lower for the AAD cohort versus the cohort without AAD $(P<0.05$ for both). However, pharmacy costs were similar between the cohorts during the baseline period $(P=0.825)$. 


\begin{tabular}{|c|c|c|c|}
\hline TABLE 2 & \multicolumn{3}{|c|}{$\begin{array}{l}\text { All-Cause Health Care Costs During } \\
\text { 12-Month Follow-Up Period } \\
\text { (2012 U.S. Dollars) }\end{array}$} \\
\hline & With AAD & Without AAD & \\
\hline $\begin{array}{l}\text { Unadjusted } \\
\text { Costs (\$) }\end{array}$ & Mean (SD) & Mean (SD) & $P$ Value \\
\hline Total & $\begin{array}{c}31,991 \\
(39,341) \\
\end{array}$ & $\begin{array}{l}25,395 \\
(36,414)\end{array}$ & $<0.001$ \\
\hline Medical & $\begin{array}{c}28,429 \\
(38,398) \\
\end{array}$ & $\begin{array}{c}22,082 \\
(35,569) \\
\end{array}$ & $<0.001$ \\
\hline Inpatient & $\begin{array}{c}14,995 \\
(30,596)\end{array}$ & $\begin{array}{c}10,261 \\
(27,649) \\
\end{array}$ & $<0.001$ \\
\hline Outpatient & $\begin{array}{c}13,434 \\
(16,412)\end{array}$ & $\begin{array}{c}11,821 \\
(17,171) \\
\end{array}$ & 0.011 \\
\hline Prescription & $\begin{array}{c}3,562 \\
(4,935) \\
\end{array}$ & $\begin{array}{c}3,312 \\
(5,387) \\
\end{array}$ & 0.212 \\
\hline $\begin{array}{l}\text { Adjusted } \\
\text { Costs }^{\mathrm{a}}(\$)\end{array}$ & Predicted Mean & Predicted Mean & $\begin{array}{l}\text { Difference } \\
(95 \% \mathrm{CI})\end{array}$ \\
\hline Total & 31,227 & 25,491 & $\begin{array}{c}5,737 \\
(1,897-10,116)\end{array}$ \\
\hline Medical & 29,116 & 22,126 & $\begin{array}{c}6,990 \\
(2,974-11,648) \\
\end{array}$ \\
\hline Inpatient & 15,496 & 10,253 & $\begin{array}{c}5,243 \\
(2,182-9,058) \\
\end{array}$ \\
\hline Outpatient & 13,990 & 11,816 & $\begin{array}{c}2,174 \\
(561-3,998)\end{array}$ \\
\hline Prescription & 3,731 & 3,347 & $\begin{array}{c}384 \\
(120-668) \\
\end{array}$ \\
\hline
\end{tabular}

Note: Bold font indicates $P<0.05$ and statistical significance.

${ }^{a}$ Each cost component is estimated from separate regression models and represents a predicted rather than an observed value. Therefore, prescription and medical cost components may not add up to the total. Similarly, inpatient and outpatient may not add up to the total medical cost.

$A A D=$ alcohol abuse or dependence; $C I=$ confidence interval; $S D=$ standard deviation

\section{Outcomes}

In this study, an AAD diagnosis in long-term CNCP opioid users was associated with higher rates of all assessed adverse outcomes, including opioid overdose, accident, and injury (Figure 3). Although opioid overdose and accidents were uncommon, the study cohort with diagnosed AAD had 5 times the proportion of patients with opioid overdose than the cohort without AAD ( $1 \%$ vs. $0.2 \%$, respectively; $P<0.001)$ and 2.3 times the proportion of patients with accidents ( $7 \%$ vs. $3 \%$, respectively; $P<0.001$ ). Injuries were also more common, with 1.2 times the proportion of patients with AAD experiencing injuries requiring interaction with the health care system compared with those without diagnosed AAD (46\% vs. 37\%, respectively; $P<0.001)$. After adjusting for baseline covariates, the AAD cohort had a 32\% significantly higher odds of injuries compared with those without AAD (odds ratio $[\mathrm{OR}]=1.32$; $P=0.035$; Figure 3). The difference in accidents, however, became nonsignificant after adjusting for covariates ( $\mathrm{OR}=1.36$; $P=0.227$ ). No multivariable analyses were conducted for opioid overdose due to a small sample size.
In the 12-month follow-up period, total all-cause health care costs (Table 2) were 26\% higher for those with AAD than for those without AAD (mean $\$ 31,991$ vs. $\$ 25,395$, respectively; $P<0.001$ ). This was driven by $46 \%$ higher inpatient costs (mean $\$ 14,995$ with AAD vs. $\$ 10,261$ without AAD; $P<0.001$ ). Inpatient costs were $47 \%$ of total health care costs among patients with AAD compared with $40 \%$ among those without AAD. Outpatient medical costs were \$1,613 higher among patients with AAD versus patients without (mean $\$ 13,343$ vs. $\$ 11,821$, respectively; $P=0.011$, while prescription costs were similar $(P=0.212)$. Similar results were noted after adjusting for differences in baseline covariates for all-cause health care costs and inpatient and outpatient medical costs. However, the difference in prescription costs was also statistically significant.

\section{Discussion}

In this analysis, 3.5\% of long-term opioid users had a diagnosis of AAD before opioid initiation. This is half of the estimated prevalence of AAD in the general population (7\% in 2013) and significantly lower than earlier estimates in chronic pain patients who are on long-term opioid therapy (31\%-46\%).11,12 The lower estimate of AAD prevalence in this study is likely because of the definition used and the nature of the available data. This study used $\geq 1$ medical claim with a diagnosis of AAD compared with the use of patient reports and/or electronic medical records in other studies. Claims-based studies may underestimate rates of AAD for several reasons, including poor recognition of AAD in medical practice and disincentives for patients to disclose these disorders and for physicians to render these diagnoses (including concerns over reimbursement for related medical care, malpractice, or regulatory scrutiny). It is also possible that patients with AAD are more likely to be uninsured.

With respect to the adverse outcomes, this study revealed higher rates of opioid overdose, accident, and injury in longterm opioid chronic pain patients with a history of AAD. Although rates of overdose were low, $1 \%$ of AAD patients experienced such an adverse outcome compared with only $0.2 \%$ of patients without AAD. However, these results must be interpreted with caution because of the relatively small sample size. Nevertheless, future studies are needed to further examine the relationship of opioid overdose and AAD. AAD patients were also more likely to experience accidents or injuries requiring medical attention. These data suggest an increased risk of adverse outcomes among patients with AAD receiving longterm opioid therapy.

Previous studies also suggest that a history of AAD is associated with increased rates of opioid abuse behaviors such as "doctor shopping," diversion, and prescription forgery, ${ }^{5,12-14}$ This study could not assess these aberrant behaviors specifically, since this information was not available in the claims database. However, given the increased rate of adverse 
outcomes such as injuries, accidents, and opioid overdoses, there is some indication of increased potential of abuse among $\mathrm{CNCP}$ opioid users with a history of AAD. In addition, the rate of opioid prescribing in general has increased substantially over the last 3 decades and even more so among patients with a history of substance abuse disorder. ${ }^{15-19}$ Although we saw a decrease in the rate of AAD over the 5 years of the study, this may not be a significant decrease, and CNCP patients with a history of AAD will likely continue to contribute significantly to the economic burden.

Overall, alcohol abuse contributes $\$ 223.5$ billion in economic burden annually in the United States (2006 U.S. dollars). ${ }^{20}$ Thus, these patients have the potential to use considerable health care resources, as demonstrated in this study. In the 12 months before CNCP diagnosis, the cohort of patients without AAD had greater total $(\$ 2,284)$ and medical costs $(\$ 2,312)$ relative to those with AAD. However, following CNCP diagnosis and opioid initiation, costs were significantly higher by $21 \%$ in the AAD cohort than the non-AAD cohort in the 12 months following opioid initiation.

CNCP patients with a history of alcohol abuse and pain refractory to nonopioid treatment options may need adequate pain-related treatment options, and opioids may be a necessary treatment option. This study found that patients with $\mathrm{AAD}$, when prescribed long-term opioids, have higher risks for adverse events such as injuries than those patients without AAD. The risk-benefit ratio of opioid therapy should be carefully considered in patients with AAD, and if used, these patients should be monitored closely. If the risks outweigh the benefits at any point during therapy, opioid therapy should be discontinued. There are several ways payers can manage opioid abuse risk, including encouraging the use of risk management strategies among health care providers. ${ }^{21}$ As hypothesized, but not previously described, the risks of using opioids in CNCP patients with a history of alcohol abuse, if not managed appropriately, may translate into increased costs.

\section{Limitations}

These results need to be evaluated in light of the study limitations. Claims data are not collected for research purposes per se, so the conclusions drawn from such data are limited by the accuracy and completeness of the claims submitted. Diagnoses of AAD may be underreported (i.e., not coded on medical claims), likely leading to misclassification of study cohorts. However, misclassification will only result in dilution of the observed effects, so the differences between cohorts may be underestimated. Survival bias likely underestimated the rates of adverse outcomes, especially opioid overdose. Patients who died as a result of an adverse outcome or for other reasons were excluded, since all patients were required to have 12 months of continuous enrollment following opioid initiation. Finally, there are inherent limitations in using prescription claims to measure opioid use, since filling a prescription may not be indicative of actual usage.

\section{Conclusions}

Following the first year after opioid initiation, CNCP patients with long-term opioid use and diagnosed with AAD had 5 times the proportion of opioid overdose, 2.3 times the proportion of accidents, and 1.2 times the proportion of injuries compared with CNCP patients with long-term opioid use and no diagnosed AAD. Long-term CNCP patients with AAD also had higher total health care costs than those without AAD. Further research is necessary to determine if there is a dosedependent relationship between alcohol use and opioid intake with adverse outcomes. Future research may also investigate the prevalence of simultaneous use of alcohol with opioids and if the observed differences in adverse outcomes between patients with and without AAD dependence are influenced solely by the use of alcohol or represent a synergistic effect between alcohol and opioid use. The results of this study indicate that the risks of treating patients with long-term opioids must be carefully considered, especially in patients with a history of AAD, which is associated with increased risks of overdoses, accidents, and other injuries.

\section{Authors}

PAMELA B. LANDSMAN-BLUMBERG, MPH, DrPH, and ANNA D. COUTINHO, BPharm, PhD, Xcenda, Palm Harbor, Florida. KAVITA GAJRIA, MS; PAUL P. YEUNG, MD, MPH; and RICHARD WHITE, PhD, MPH, MBE, Teva Pharmaceuticals, Frazer, Pennsylvania. NATHANIEL KATZ, MD, MS, Analgesic Solutions, Natick, Massachusetts.

AUTHOR CORRESPONDENCE: Pamela B. Landsman-Blumberg, MPH, DrPH, Xcenda, 4114 Woodlands Pkwy., Ste. 500, Palm Harbor, FL 34685. E-mail: Pamela.Blumberg@xcenda.com

\section{DISCLOSURES}

Funding for this research study and resultant publication was provided by Teva Global Health Economics and Outcomes Research, which fully reviewed the manuscript. Gajria and Yeung are employees of Teva Pharmaceuticals. White was an employee of Teva Pharmaceuticals at the time this research was conducted. Blumberg and Coutinho are employees of Xcenda, which received research funding from Teva Pharmaceuticals for the conduct of this study and for the preparation of this manuscript. Katz has received research funding and consulting fees from Teva Pharmaceuticals unrelated to this study.

Study concept and design were contributed by Katz, White, and Blumberg, along with Coutinho and Yeung. Coutinho took the lead in data collection, assisted by the other authors. Data interpretation was performed by Blumberg, Katz, and Gajria, along with the other authors. The manuscript was written by Gajria, Yeung, Coutinho, and Blumberg, along with Katz and White, and revised by Gajria, Blumberg, Katz, and Coutinho, along with Yeung and White 


\section{ACKNOWLEDGMENTS}

Bethany Sawchyn provided writing support for the manuscript. Sham Chaudhari provided assistance with preparing the analytic dataset. Editorial assistance was provided by Peloton Advantage and funded by Teva Pharmaceuticals.

\section{REFERENCES}

1. Morasco BJ, Gritzner S, Lewis L, Oldham R, Turk DC, Dobscha SK. Systematic review of prevalence, correlates, and treatment outcomes for chronic non-cancer pain in patients with comorbid substance use disorder. Pain. 2011;152(3):488-97.

2. Edlund MJ, Sullivan M, Steffick D, Harris KM, Wells KB. Do users of regularly prescribed opioids have higher rates of substance use problems than nonusers? Pain Med. 2007;8(8):647-56.

3. Edlund MJ, Austen MA, Sullivan MD, et al. Patterns of opioid use for chronic noncancer pain in the Veterans Health Administration from 2009 to 2011. Pain. 2014;155(11):2337-43

4. Leider HL, Dhaliwal J, Davis EJ, Kulakodlu M, Buikema AR. Healthcare costs and nonadherence among chronic opioid users. Am J Manag Care. 2011;17(1):32-40

5. Chou R, Fanciullo GJ, Fine PG, et al; American Pain Society-American Academy of Pain Medicine Opioids Guidelines Panel. Clinical guidelines for the use of chronic opioid therapy in chronic noncancer pain. J Pain. 2009; 10(2):113-30.

6. Zacny JP, Gutierrez S. Subjective, psychomotor, and physiological effects of oxycodone alone and in combination with ethanol in healthy volunteers. Psychopharmacology (Berl). 2011;218(3):471-81.

7. Manchikanti L, Abdi S, Atluri S, et al; American Society of Interventional Pain Physicians. American Society of Interventional Pain Physicians (ASIPP) guidelines for responsible opioid prescribing in chronic non-cancer pain: part 2-guidance. Pain Physician. 2012;15(3 Suppl):S67-S16.

8. Weisner CM, Campbell CI, Ray GT, et al. Trends in prescribed opioid therapy for non-cancer pain for individuals with prior substance use disorders. Pain. 2009;145(3):287-93.

9. Dunbar SA, Katz NP. Chronic opioid therapy for nonmalignant pain in patients with a history of substance abuse: report of 20 cases. J Pain Symptom Manage. 1996;11(3):163-71.
10. Charlson ME, Pompei P, Ales KL, MacKenzie CR. A new method of classifying prognostic comorbidity in longitudinal studies: development and validation. J Chronic Dis. 1987;40(5):373-83.

11. Substance Abuse and Mental Health Services Administration. 2013 National Survey on Drug Use and Health (NSDUH). Table 5.8BSubstance dependence or abuse in the past year among persons aged 18 or older, by demographic characteristics: percentages, 2012 and 2013. Available at: http://www.samhsa.gov/data/sites/default/ files/NSDUH-DetTabsPDFWHTML2013/Web/HTML/NSDUHDetTabsSect5peTabslto56-2013.htm\#tab5.8b. Accessed May 26, 2017.

12. Reid MC, Engles-Horton LL, Weber MB, Kerns RD, Rogers EL, O'Connor PG. Use of opioid medications for chronic noncancer pain syndromes in primary care. J Gen Intern Med. 2002;17(3):173-79.

13. Ives TJ, Chelminski PR, Hammett-Stabler CA, et al. Predictors of opioid misuse in patients with chronic pain: a prospective cohort study. BMC Health Serv Res. 2006;6:46.

14. Michna E, Ross EL, Hynes WL, et al. Predicting aberrant drug behavior in patients treated for chronic pain: importance of abuse history. J Pain Symptom Manage. 2004;28(3):250-58.

15. Caudill-Slosberg MA, Schwartz LM, Woloshin S. Office visits and analgesic prescriptions for musculoskeletal pain in U.S.: 1980 vs. 2000. Pain. 2004;109(3):514-19.

16. Edlund MJ, Martin BC, Devries A, Fan MY, Braden JB, Sullivan MD. Trends in use of opioids for chronic noncancer pain among individuals with mental health and substance use disorders: the TROUP study. Clin J Pain. 2010;26(1):1-8.

17. Kenan K, Mack K, Paulozzi L. Trends in prescriptions for oxycodone and other commonly used opioids in the United States, 2000-2010. Open Med. 2012;6(2):e4l-e47.

18. Larochelle MR, Zhang F, Ross-Degnan D, Wharam JF. Trends in opioid prescribing and co-prescribing of sedative hypnotics for acute and chronic musculoskeletal pain: 2001-2010. Pharmacoepidemiol Drug Saf. 2015;24(8):885-92.

19. Thielke SM, Simoni-Wastila L, Edlund MJ, et al. Age and sex trends in long-term opioid use in two large American health systems between 2000 and 2005. Pain Med. 2010;11(2):248-56.

20. Bouchery EE, Harwood HJ, Sacks JJ, Simon CJ, Brewer RD. Economic costs of excessive alcohol consumption in the U.S., 2006. Am J Prev Med. 2011;41(5):516-24.

21. Katz NP, Birnbaum H, Brennan MJ, et al. Prescription opioid abuse: challenges and opportunities for payers. Am J Manag Care. 2013;19(4):295-302. 


\section{APPENDIX Study Attrition}

$\geq 3$ nondiagnostic pain claims of the same type during enrollment period

$\mathrm{N}=7,760,281(\mathbf{1 0 0 . 0} \%)$

No chronic pain diagnosis in the 12 months before first pain diagnosis date

$n=6,939,279(89.4 \%)$

No diagnosis of malignancy any time in the 12 months before or any time after the first pain diagnosis date

$n=6,163,401(79.4 \%)$

Aged 18 years at first pain diagnosis date

$n=5,816,132(74.9 \%)$

No evidence of opioid treatment in the 12 months before the first pain diagnosis date

$n=4,802,240(61.9 \%)$

Continuous enrollment in medical and pharmacy coverage 12 months before the first pain diagnosis date

$n=1,828,747(23.6 \%)$

Continuous enrollment in medical and pharmacy coverage 12 months after the index date

$n=1,279,590(16.5 \%)$

Have opioid use after first diagnosis date

$n=479,079(6.2 \%)$

Final sample size: long-term opioid users

$n=21,203$ 